\title{
Skyrme interaction to second order in nuclear matter 1
}

\author{
N. Kaiser \\ Physik-Department T39, Technische Universität München, D-85747 Garching, Germany
}

\begin{abstract}
Based on the phenomenological Skyrme interaction various density-dependent nuclear matter quantities are calculated up to second order in many-body perturbation theory. The spin-orbit term as well as two tensor terms contribute at second order to the energy per particle. The simultaneous calculation of the isotropic Fermi-liquid parameters provides a rigorous check through the validity of the Landau relations. It is found that published results for these second order contributions are incorrect in most cases. In particular, interference terms between $s$-wave and $p$-wave components of the interaction can contribute only to (isospin or spin) asymmetry energies. Even with nine adjustable parameters, one does not obtain a good description of the empirical nuclear matter saturation curve in the low density region $0<\rho<2 \rho_{0}$. The reason for this feature is the too strong density-dependence $\rho^{8 / 3}$ of several second-order contributions. The inclusion of the density-dependent term $\frac{1}{6} t_{3} \rho^{1 / 6}$ is therefore indispensable for a realistic description of nuclear matter in the Skyrme framework.
\end{abstract}

\section{Introduction and summary}

Mean-field theories are widely used to analyze a large variety of many-body systems for which the independent particle picture can be applied. In nuclear physics, mean-field approaches generally lead to satisfactory results for bulk properties of atomic nuclei, such as their masses, radii and ground-state deformations. However, in some cases improvements beyond the mean-field treatment are definitely needed. For instance, mean-field models do not predict accurately the single-particle spectra (i.e. energies, spectroscopic factors and fragmentations of single-particle states), especially close to the Fermi energy. A possibility for improvement is given by introducing the coupling between individual nucleon degrees of freedom and collective degrees of freedom.

Phenomenological Skyrme forces have been used widely and successfully for non-relativistic nuclear structure calculations of medium-mass and heavy nuclei [1, 2]. In effect such purely phenomenological two-body interactions can be viewed as to provide a convenient parameterization of the nuclear energy density functional on which the self-consistent mean-field treatment (in the form of solving the Kohn-Sham equations) can be based. In order to go beyond the common mean-field (Hartree-Fock) approximation, second-order calculations with the phenomenological Skyrme force have been pursued in recent works by Moghrabi, Grasso et al. [3, 4, 5, 6]. These exploratory studies have been concerned with the second-order contributions to the equation of state of infinite isospin-symmetric nuclear matter and pure neutron matter. Since the (two-body) Skyrme interaction is of zero range, divergences occur at second order in many-body perturbation theory, which have been treated either by cutoff regularization [3, 4] or by dimensional regularization [5. It has been concluded in refs. 4, 5] that after readjusting the parameters of the Skyrme interaction a simultaneous good reproduction of the equation of state of isospin-symmetric and isospin-asymmetric nuclear matter can be achieved at second order.

However, a closer inspection of the published expressions for the second-order contributions in ref. [5] raises doubts about their completeness and correctness. The first deficit is the omission of a second-order contribution from the spin-orbit term proportional to $W_{0}$. This $p$-wave interaction

\footnotetext{
${ }^{1}$ This work has been supported in part by DFG and NSFC (CRC110).
} 
term averages to zero in infinite matter at first order, but not at second order (see e.g. appendix A in ref. [7] for the second-order contributions from a "boson-exchange" spin-orbit interaction). Secondly, the stated expressions [5] for the energy per particle of isospin-symmetric nuclear matter and pure neutron matter include interference terms between $s$-wave and $p$-wave components of the Skyrme interaction (i.e. terms proportional to $t_{0} t_{2}$ and $t_{1} t_{2}$ ). It is very questionable how such a mixing of different parities could be induced by the (homogeneous) nuclear medium. Moreover, the expressions for the cutoff-independent second-order contributions given in a later paper [6] by the same authors disagree partly with those published in ref. [5].

These apparent inconsistencies provide good reason to reconsider and extend the calculation of the Skyrme interaction to second order in nuclear matter. The present paper is organized as follows: In section 2, the Skyrme force is introduced as a Galilei-invariant two-nucleon contactinteraction in momentum space. On this basis, first-order Hartree-Fock results are rederived for a variety of density-dependent nuclear matter quantities, such as the energy per particle and the (isospin and spin) asymmetry energy. Section 3 is devoted to the second-order calculation in manybody perturbation theory. We employ a scheme where the in-medium (particle-hole) propagator is decomposed into the vacuum propagator and a medium-insertion. This scheme has the advantage that ultraviolet divergences appear only in the second-order Hartree and Fock diagrams with two medium-insertions. The pertinent one-loop rescattering in vacuum is treated by dimensional regularization and shown to vanish identically. As a consequence the genuine second-order terms arise entirely from the Hartree and Fock diagrams with three medium-insertions. The occurring finite integrals over three Fermi spheres can be solved analytically and the results for the nuclear matter quantities consist of even powers of the Fermi momentum $k_{f}$ multiplied with products of the Skyrme parameters and a numerical coefficient of the form $\ln 2+r_{j}$, where $r_{j}$ is some rational number. It is found that the energy per particle of isospin-symmetric nuclear matter $\bar{E}\left(k_{f}\right)$ and pure neutron matter $\bar{E}_{n}\left(k_{n}\right)$ do not include any $s$-wave and $p$-wave interference terms at second order. However, such $s p$-interference terms show up in the (isospin and spin) asymmetry energies. The reason and condition for $s p$-mixing to emerge is explained in appendix A by considering the in-medium loop for a proton-neutron pair in nuclear matter with unequal proton and neutron densities. In section 4, the effective nucleon mass and the isotropic Fermi-liquid parameters are calculated up to second order. These results allow for a rigorous check through the validity of several Landau relations [8]. In section 5, the effects of tensor interactions at second order are computed. Actually, with the inclusion of two independent tensor terms the Skyrme force gets completed to the most general two-nucleon contact-interaction quadratic in momenta. In section 6 , the iteration of the Skyrme force with the one-photon exchange is considered. This mechanism generates a charge-symmetry breaking contribution to the energy density of protons, which could help to resolve the Nolen-Schiffer anomaly [9]. This anomaly refers to the observation that the binding energy differences of mirror nuclei cannot be explained by the Coulomb interaction of the protons alone. Remarkably, one finds an infrared-finite result for the energy per proton $\bar{E}_{p}\left(k_{p}\right)$ even though a regulator photon mass $m_{\gamma}$ has be introduced initially. Section 7 deals with some provisional parameter fits. It is found that with nine adjustable parameters in the complete second-order expression, one does not obtain a good reproduction of a realistic nuclear matter saturation curve in the low-density region $0<\rho<2 \rho_{0}$. The reason for this failure lies in the too strong density-dependence $\rho^{8 / 3}$ of several second-order contributions. From this observation one can conclude that the inclusion of ( ad hoc) density-dependent terms is indispensable for a realistic description of nuclear matter in the Skyrme approach. At a formal level, these are taken into account by performing appropriate parameter substitutions, $t_{0} \rightarrow t_{0}+\frac{1}{6} t_{3} \rho^{1 / 6}$ and $t_{0} x_{0} \rightarrow t_{0} x_{0}+\frac{1}{6} t_{3} x_{3} \rho^{1 / 6}$, in all first- and second-order expressions derived in this work.

Let us clarify that the main purpose of this paper is to compile the (correct) analytical expressions for various nuclear matter quantities as they arise from a second-order calculation with 
a two-body contact-interaction of the Skyrme type. The question of whether improvements are possible in comparison to a first-order mean-field approach has to be explored in future studies.

\section{First-order Hartree-Fock calculation}

This section serves mostly for orientation. We introduce various density-dependent nuclear matter quantities and specify their first-order Hartree-Fock contributions. The Skyrme interaction is a zero-range two-nucleon contact-interaction with transition matrix-element in momentum-space:

$$
V_{\mathrm{Sk}}=t_{0}\left(1+x_{0} P_{\sigma}\right)+\frac{t_{1}}{2}\left(1+x_{1} P_{\sigma}\right)\left(\vec{q}_{\mathrm{out}}^{2}+\vec{q}_{\mathrm{in}}^{2}\right)+t_{2}\left(1+x_{2} P_{\sigma}\right) \vec{q}_{\mathrm{out}} \cdot \vec{q}_{\mathrm{in}}+i W_{0}\left(\vec{\sigma}_{1}+\vec{\sigma}_{2}\right) \cdot\left(\vec{q}_{\mathrm{out}} \times \vec{q}_{\mathrm{in}}\right) .
$$

Here, $P_{\sigma}=\left(1+\vec{\sigma}_{1} \cdot \vec{\sigma}_{2}\right) / 2$ is the spin-exchange operator and $\vec{q}_{\text {in }}=\left(\vec{p}_{1}-\vec{p}_{2}\right) / 2$ and $\vec{q}_{\text {out }}=\left(\vec{p}_{1}^{\prime}-\vec{p}_{2}^{\prime}\right) / 2$ denote half of the momentum difference in the initial and final state, respectively. This particular form of $V_{\mathrm{Sk}}$, involving only external momentum differences, is dictated by Galilei invariance (and invariance under time-reversal: $\vec{q}_{\text {in }} \leftrightarrow-\vec{q}_{\text {out }}, \vec{\sigma}_{1,2} \rightarrow-\vec{\sigma}_{1,2}$ ). The individual terms in eq.(1) stand for: $s$-wave contact interaction $\left(t_{0}\right), s$-wave effective range correction $\left(t_{1}\right), p$-wave contact interaction $\left(t_{2}\right)$, and spin-orbit interaction $\left(W_{0}\right)$. The general contact-potential up to order $p^{2}$, as it is commonly used in chiral effective field theory [10, 11, 12], has two additional tensor terms which will be studied separately in section 5. Note that we have not explicitly written in eq.(1) the density-dependent Skyrme term $\frac{1}{6} t_{3}\left(1+x_{3} P_{\sigma}\right) \rho^{1 / 6}$. Formally, it can be included through the substitution of parameters $t_{0} \rightarrow t_{0}+\frac{1}{6} t_{3} \rho^{1 / 6}$ and $t_{0} x_{0} \rightarrow t_{0} x_{0}+\frac{1}{6} t_{3} x_{3} \rho^{1 / 6}$ in all following formulas. However, from a rigorous point of view such density-dependent couplings will lead to a conflict with basic many-body relations (Landau relations [8] and Hugenholtz-Van-Howe theorem [13]), whenever derivatives with respect to density $\rho$ are involved.
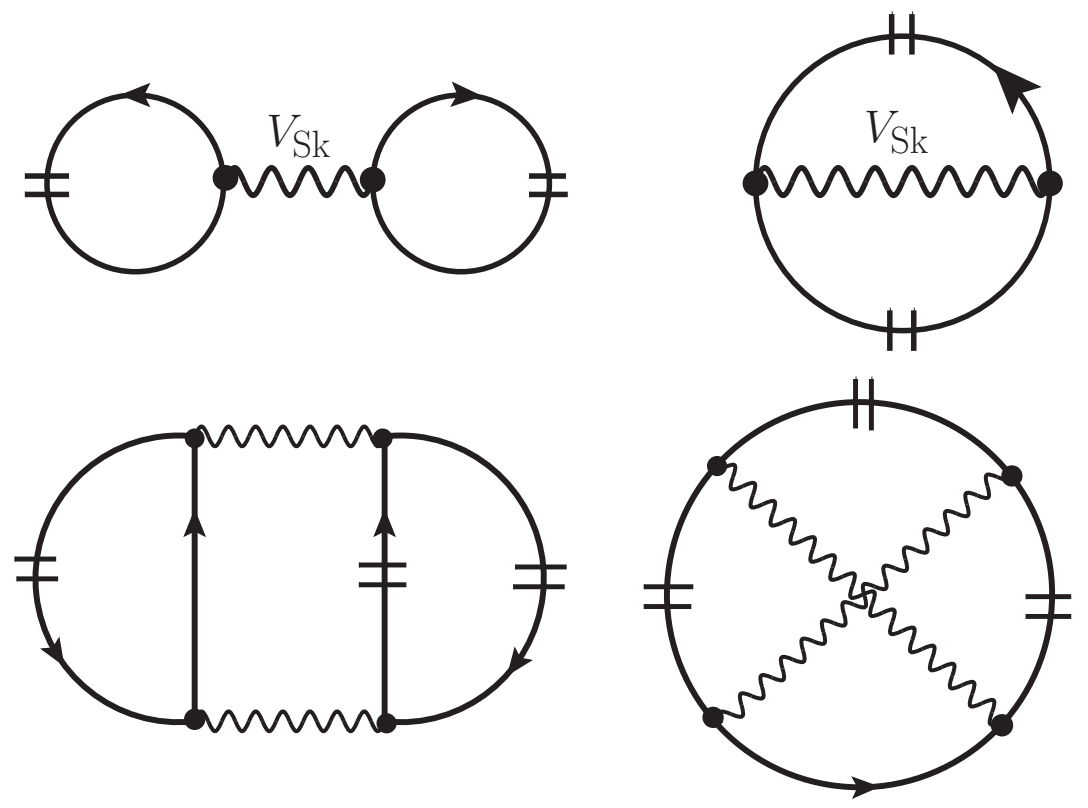

Figure 1: Upper row: First-order Hartree and Fock diagrams with two medium-insertions and combinatoric factor $1 / 2$. The wavy line represents the Skyrme interaction $V_{\mathrm{Sk}}$. Lower row: Secondorder Hartree and Fock diagrams with three medium-insertions and combinatoric factor 1.

The first-order Hartree and Fock diagrams generated by $V_{\mathrm{Sk}}$ are shown in the upper part of Fig. 1. The short double-line symbolizes the medium-insertion (i.e. the difference between the inmedium and vacuum nucleon-propagator) proportional to the step-function $\theta\left(k_{f}-\left|\vec{p}_{j}\right|\right)$. The Fermi 
momentum $k_{f}$ is related the (total) nucleon density in the usual way, $\rho=2 k_{f}^{3} / 3 \pi^{2}$. Performing the appropriate spin- and isospin-traces and integrals over two Fermi spheres, one obtains for the energy per particle of isospin-symmetric nuclear matter the well-known first-order expression:

$$
\bar{E}\left(k_{f}\right)^{(1 \mathrm{st})}=\frac{k_{f}^{3}}{4 \pi^{2}}\left\{t_{0}+\frac{k_{f}^{2}}{10}\left[3 t_{1}+t_{2}\left(5+4 x_{2}\right)\right]\right\} .
$$

In the same way, but leaving out the isospin-trace, the energy per particle of pure neutron matter comes out as:

$$
\bar{E}_{n}\left(k_{n}\right)^{(1 \mathrm{st})}=\frac{k_{n}^{3}}{4 \pi^{2}}\left\{\frac{t_{0}}{3}\left(1-x_{0}\right)+\frac{k_{n}^{2}}{10}\left[t_{1}\left(1-x_{1}\right)+3 t_{2}\left(1+x_{2}\right)\right]\right\}
$$

with $k_{n}$ the neutron Fermi momentum related to the neutron density by $\rho_{n}=k_{n}^{3} / 3 \pi^{2}$. Another important quantity of high interest is the isospin asymmetry energy of nuclear matter. An isospinasymmetric configuration with unequal proton and neutron Fermi momenta $k_{p, n}=k_{f}(1 \mp \delta)^{1 / 3}$ is realized by the substitution:

$$
\theta\left(k_{f}-\left|\vec{p}_{j}\right|\right) \rightarrow \frac{1+\tau_{3}}{2} \theta\left(k_{p}-\left|\vec{p}_{j}\right|\right)+\frac{1-\tau_{3}}{2} \theta\left(k_{n}-\left|\vec{p}_{j}\right|\right),
$$

for the medium-insertion and an additional expansion of the (asymmetric) energy per particle:

$$
\bar{E}_{\text {as }}\left(k_{p}, k_{n}\right)=\bar{E}\left(k_{f}\right)+\delta^{2} A\left(k_{f}\right)+\mathcal{O}\left(\delta^{4}\right),
$$

around $\delta=0$ defines the density-dependent isospin asymmetry $A\left(k_{f}\right)$ of nuclear matter. The first-order Hartree-Fock contribution from the Skyrme interaction $V_{\mathrm{Sk}}$ has the well-known form:

$$
A\left(k_{f}\right)^{(1 \mathrm{st})}=\frac{k_{f}^{3}}{12 \pi^{2}}\left\{-t_{0}\left(1+2 x_{0}\right)+\frac{k_{f}^{2}}{3}\left[t_{2}\left(4+5 x_{2}\right)-3 t_{1} x_{1}\right]\right\} .
$$

In an analogous way the density-dependent spin asymmetry energy $S\left(k_{f}\right)$ of nuclear matter can be constructed. The substitution in spin-space:

$$
\theta\left(k_{f}-\left|\vec{p}_{j}\right|\right) \rightarrow \frac{1+\sigma_{3}}{2} \theta\left(k_{\uparrow}-\left|\vec{p}_{j}\right|\right)+\frac{1-\sigma_{3}}{2} \theta\left(k_{\downarrow}-\left|\vec{p}_{j}\right|\right),
$$

with $k_{\uparrow, \downarrow}=k_{f}(1 \pm \eta)^{1 / 3}$ the Fermi momenta of the spin-up and spin-down nucleons, is followed by an expansion of the energy per particle of spin-polarized nuclear matter around $\eta=0$ :

$$
\bar{E}_{\mathrm{pol}}\left(k_{\uparrow}, k_{\downarrow}\right)=\bar{E}\left(k_{f}\right)+\eta^{2} S\left(k_{f}\right)+\mathcal{O}\left(\eta^{4}\right) .
$$

In the absence of any quantitative empirical knowledge, the minimal condition to be satisfied by the spin asymmetry energy is that it must be positive: $S\left(k_{f}\right)>0$. This guarantees the spinstability of nuclear matter by excluding a phase transition to a ferromagnetic state of lower energy density. The first-order Hartree-Fock contribution to $S\left(k_{f}\right)$ from the Skyrme interaction $V_{\text {Sk }}$ reads:

$$
S\left(k_{f}\right)^{(1 \mathrm{st})}=\frac{k_{f}^{3}}{12 \pi^{2}}\left\{t_{0}\left(2 x_{0}-1\right)+\frac{k_{f}^{2}}{3}\left[3 t_{1} x_{1}+t_{2}\left(4+5 x_{2}\right)\right]\right\} .
$$

One notices that the expression for $S\left(k_{f}\right)^{(1 \mathrm{st})}$ can be obtained from $A\left(k_{f}\right)^{(1 \mathrm{st})}$ in eq.(6) by just changing the signs of the two parameters $x_{0}$ and $x_{1}$. The reason behind this connection is an equivalent form of the contact-interaction which results from $V_{\mathrm{Sk}}$ in eq.(1) through the substitutions:

$$
\begin{aligned}
& t_{0}\left(1+x_{0} P_{\sigma}\right) \rightarrow t_{0}\left(1-x_{0} P_{\tau}\right) \\
& t_{1}\left(1+x_{1} P_{\sigma}\right) \rightarrow t_{1}\left(1-x_{1} P_{\tau}\right) \\
& t_{2}\left(1+x_{2} P_{\sigma}\right) \rightarrow t_{2}\left(1+x_{2} P_{\tau}\right)
\end{aligned}
$$


with $P_{\tau}=\left(1+\vec{\tau}_{1} \cdot \vec{\tau}_{2}\right) / 2$ the isospin-exchange operator. The known eigenvalues of $P_{\sigma}$ and $P_{\tau}$ are $(-1)^{S+1}$ and $(-1)^{I+1}$ with $S, I \in\{0,1\}$ the total spin and isospin quantum numbers. Since the parameters $t_{0}$ and $t_{1}$ act only in $s$-wave states with $S+I=1$, one has effectively for $s$-waves the equality $P_{\sigma}=-P_{\tau}$. Likewise, the parameter $t_{2}$ acts only in $p$-wave states with $S+I=0,2$ and therefore for $p$-waves the opposite equality $P_{\sigma}=P_{\tau}$ holds. Another obvious consequence of the equivalent interaction is that only the combinations $t_{0}\left(1-x_{0}\right), t_{1}\left(1-x_{1}\right)$ and $t_{2}\left(1+x_{2}\right)$ can contribute in neutron matter, since $P_{\tau}=1$ for a two-neutron state. This is exemplified by calculating the first-order contribution to the spin asymmetry energy of pure neutron matter:

$$
S_{n}\left(k_{n}\right)^{(1 \mathrm{st})}=\frac{k_{n}^{3}}{12 \pi^{2}}\left\{t_{0}\left(x_{0}-1\right)+\frac{k_{n}^{2}}{3}\left[t_{1}\left(x_{1}-1\right)+3 t_{2}\left(1+x_{2}\right)\right]\right\} .
$$

Again, the density-dependent quantity $S_{n}\left(k_{n}\right)$ must be positive: $S_{n}\left(k_{n}\right)>0$, in order to prevent neutron matter from a ferromagnetic instability at low and moderate densities. We conclude this section by giving the contributions from the relativistically improved kinetic energy:

$$
\begin{gathered}
\bar{E}\left(k_{f}\right)^{(\mathrm{kin})}=\frac{3 k_{f}^{2}}{10 M}-\frac{3 k_{f}^{4}}{56 M^{3}}, \quad \bar{E}_{n}\left(k_{n}\right)^{(\mathrm{kin})}=\frac{3 k_{n}^{2}}{10 M}-\frac{3 k_{n}^{4}}{56 M^{3}}, \\
A\left(k_{f}\right)^{(\mathrm{kin})}=S\left(k_{f}\right)^{(\mathrm{kin})}=\frac{k_{f}^{2}}{6 M}-\frac{k_{f}^{4}}{12 M^{3}}, \quad S_{n}\left(k_{n}\right)^{(\mathrm{kin})}=\frac{k_{n}^{2}}{6 M}-\frac{k_{n}^{4}}{12 M^{3}},
\end{gathered}
$$

where $M=939 \mathrm{MeV}$ denotes the average nucleon mass.

\section{Second-order Hartree-Fock calculation}

In this section we derive analytical expressions for the contributions to the introduced nuclear matter quantities as they arise from a second-order calculation with the Skyrme interaction $V_{\mathrm{Sk}}$. At second order in many-body perturbation theory the energy density is represented by closed three-loop Hartree and Fock diagrams with either two or three medium-insertions. The first set involves the rescattering of two nucleons in the vacuum (i.e. without Pauli blocking corrections). For the contact-interaction $V_{\mathrm{Sk}}$ this one-loop diagram leads to principal-value integrals of the form:

$$
f \frac{d^{3} l}{(2 \pi)^{3}} \frac{M}{\vec{l}^{2}-\vec{q}^{2}}\left\{1, \vec{l}^{2},\left(\vec{l}^{2}\right)^{2},(\vec{l} \cdot \vec{q})^{2}\right\}
$$

with $\vec{q}=\left(\vec{p}_{1}-\vec{p}_{2}\right) / 2$, where $\vec{p}_{1,2}$ are two external momenta inside the Fermi sphere $\left|\vec{p}_{1,2}\right|<k_{f}$. The first factor in eq.(14) is the energy denominator and the other factors in curly brackets come from the Galilei-invariant contact-interaction. After angular integration and partial fraction decomposition of the radial integrand one gets power divergences of the form $\int_{0}^{\infty} d l\left\{1, l^{2}, l^{4}\right\}$, which are set to zero in dimensional regularization. The remaining convergent principal-value integral $f_{0}^{\infty} d l\left(l^{2}-q^{2}\right)^{-1}=0$ vanishes identically. As a side remark we note that cutoff regularization would provide additional terms $\Lambda^{1,3,5}, \Lambda^{1,3} \vec{q}^{2}$ and $\Lambda\left(\vec{q}^{2}\right)^{2}$ proportional to odd powers of the cutoff $\Lambda$. For the first two the $\Lambda$-dependence could be absorbed into the coupling constants $t_{j}$ and $t_{j} x_{j}$, while the last one would require the introduction of a four-derivative contact-interaction.

In dimensional regularization (or after renormalization) one is therefore left with the Hartree and Fock diagrams with three medium-insertions shown in the lower part of Fig. 1. These lead to (finite) principal-value integrals over three Fermi spheres of the form:

$$
\underset{\left|\vec{p}_{j}\right|<k_{f}}{f} \frac{d^{3} p_{1} d^{3} p_{2} d^{3} p_{3}}{(2 \pi)^{9}} \frac{M}{\vec{q}_{1} \cdot \vec{q}_{2}} H\left(\vec{q}_{1}^{2}, \vec{q}_{1} \cdot \vec{q}_{2}, \vec{q}_{2}^{2}\right)
$$


with $\vec{q}_{1,2}=\vec{p}_{3}-\vec{p}_{1,2}$. The first factor $M /\left(\vec{q}_{1} \cdot \vec{q}_{2}\right)$ is again the energy denominator and the function $H(\ldots)$ is a polynomial of total degree two or less in its three variables. In order to evaluate eq.(15), one eliminates $\vec{p}_{2}$ in favor of $\vec{q}_{2}$ and integrates over a shifted Fermi sphere $\left|\vec{q}_{2}-\vec{p}_{3}\right|<k_{f}$. The upper boundary for the $d q_{2}$-integration is then $p_{3} y+\sqrt{k_{f}^{2}-p_{3}^{2}+p_{3}^{2} y^{2}}$, with the directional cosine $y=\hat{q}_{2} \cdot \hat{p}_{3}$. By applying this procedure to evaluate the nine-dimensional principal-value integrals, one is able to obtain analytical results for all second-order contributions. Let us also note that for $s$-wave interactions $\sim t_{0}, t_{1}$ the Hartree and Fock diagram contribute with opposite sign, whereas for $p$-wave interactions $\sim t_{2}, W_{0}$ the Hartree and Fock diagram contribute with equal sign.

Putting all pieces together one ends up with the following expression for the second-order contribution from $V_{\mathrm{Sk}}$ to the energy per particle of isospin symmetric nuclear matter:

$$
\begin{aligned}
\bar{E}\left(k_{f}\right)^{(2 \mathrm{nd})}= & \frac{M k_{f}^{4}}{280 \pi^{4}}\left\{3 t_{0}^{2}\left(1+x_{0}^{2}\right)(11-2 \ln 2)+\frac{2 k_{f}^{2}}{9} t_{0} t_{1}\left(1+x_{0} x_{1}\right)(167-24 \ln 2)\right. \\
& +\frac{k_{f}^{4}}{396} t_{1}^{2}\left(1+x_{1}^{2}\right)(4943-564 \ln 2)+\frac{k_{f}^{4}}{1188} t_{2}^{2}\left(5+8 x_{2}+5 x_{2}^{2}\right)(1033-156 \ln 2) \\
& \left.+\frac{4 k_{f}^{4}}{99} W_{0}^{2}(631-102 \ln 2)\right\} .
\end{aligned}
$$

The first important observation one can make is that there are no interference terms between $s$-wave and $p$-wave components of the Skyrme interaction $V_{\mathrm{Sk}}$. A detailed comparison to the expressions written in eqs. $(16,17)$ of ref. [5] reveals that therein only the term proportional to $t_{0}^{2}\left(1+x_{0}^{2}\right)$ is correct. The different form $\bar{E}\left(k_{f}\right)^{(2 \mathrm{nd})}=3 M k_{f}^{4}\left(A_{0}+A_{3} k_{f}^{2}+A_{5} k_{f}^{4}\right) / 2 \pi^{4}$ given in eq. (5) of the later paper [6] by the same authors represents no improvement either. It be should noted that ref. [5] did not use the advantageous scheme of expanding in the number of medium-insertions and therefore divergent integrals of the form eq.(14) as well as finite integrals of the form eq.(15) had to be continued together to $d \neq 3$ space-dimensions. Moreover, the Galilei-invariant formulation of the interaction $V_{\mathrm{Sk}}$ in momentum-space is important.

By adding half of the (previous) Hartree contribution to the Fock contribution one arrives at the energy per particle of pure neutron matter:

$$
\begin{aligned}
\bar{E}_{n}\left(k_{n}\right)^{(2 \mathrm{nd})}= & \frac{M k_{n}^{4}}{280 \pi^{4}}\left\{t_{0}^{2}\left(1-x_{0}\right)^{2}(11-2 \ln 2)+\frac{2 k_{n}^{2}}{27} t_{0} t_{1}\left(1-x_{0}\right)\left(1-x_{1}\right)(167-24 \ln 2)\right. \\
& +\frac{k_{n}^{4}}{1188} t_{1}^{2}\left(1-x_{1}\right)^{2}(4943-564 \ln 2)+\frac{k_{n}^{4}}{396} t_{2}^{2}\left(1+x_{2}\right)^{2}(1033-156 \ln 2) \\
& \left.+\frac{8 k_{n}^{4}}{297} W_{0}^{2}(631-102 \ln 2)\right\} .
\end{aligned}
$$

As expected from the consideration of the equivalent interaction in section 2 , the second-order result $\bar{E}_{n}\left(k_{n}\right)^{(2 n d)}$ involves only the parameter combinations $t_{0}\left(1-x_{0}\right), t_{1}\left(1-x_{1}\right)$ and $t_{2}\left(1+x_{2}\right)$. The corresponding expression given in ref. [5] is correct only with respect to the term proportional to $t_{0} t_{1}\left(1-x_{0}\right)\left(1-x_{1}\right)$, and the (simplest) term proportional $t_{0}^{2}\left(1-x_{0}\right)^{2}$ misses a factor 2 therein. Note that the factor $(1033-156 \ln 2) / 385$ appears also in eq.(32) of ref.[14], where the secondorder contribution to $\bar{E}_{n}\left(k_{n}\right)$ has been extracted from the resummation to all orders of particle-hole ladder diagrams generated by a spin-independent $p$-wave contact interaction.

The second-order contribution to the isospin asymmetry energy $A\left(k_{f}\right)$ is more difficult to calculate. One has to evaluate now second derivatives with respect to $\delta$ at $\delta=0$ of principal-value integrals over three Fermi spheres with different radii $k_{p, n}=k_{f}(1 \mp \delta)^{1 / 3}$. The complete expression for the second-order contribution from the Skyrme interaction $V_{\mathrm{Sk}}$ to the isospin asymmetry energy 
of nuclear matter reads:

$$
\begin{aligned}
A\left(k_{f}\right)^{(2 \mathrm{nd})}= & \frac{M k_{f}^{4}}{60 \pi^{4}}\left\{t_{0}^{2}\left[\left(1+x_{0}^{2}\right)(1-2 \ln 2)-10 x_{0}\right]\right. \\
& +\frac{k_{f}^{2}}{42} t_{0} t_{1}\left[4\left(1+x_{0} x_{1}\right)(51-22 \ln 2)+7\left(x_{0}+x_{1}\right)(4 \ln 2-57)\right] \\
& +\frac{k_{f}^{4}}{63} t_{1}^{2}\left[\left(1+x_{1}^{2}\right)\left(\frac{1301}{6}-46 \ln 2\right)+x_{1}(48 \ln 2-607)\right] \\
& +\frac{k_{f}^{4}}{567} t_{2}^{2}\left[5\left(1+x_{2}^{2}\right)\left(\frac{655}{2}-78 \ln 2\right)+x_{2}(3187-624 \ln 2)\right] \\
& +\frac{k_{f}^{2}}{6} t_{0} t_{2}\left(2+x_{0}+x_{2}+2 x_{0} x_{2}\right)(4 \ln 2-7) \\
& +\frac{2 k_{f}^{4}}{63} t_{1} t_{2}\left(2+x_{1}+x_{2}+2 x_{1} x_{2}\right)(12 \ln 2-31) \\
& \left.+\frac{4 k_{f}^{4}}{189} W_{0}^{2}(761-132 \ln 2)\right\} .
\end{aligned}
$$

Note that the third-last and second-last contribution proportional to $t_{0} t_{2}$ and $t_{1} t_{2}$ are $s p$-mixing terms. These arise solely from the second-order Hartree diagram and they are made possible by the different radii of Fermi spheres involved. The reasons and conditions for $s p$-interference to emerge for a nuclear matter quantity are explained in appendix A by considering the in-medium loop for a proton-neutron pair in nuclear matter with unequal proton and neutron densities.

In the same way (i.e. by evaluating derivatives $\partial^{2} / \partial \eta^{2}$ at $\eta=0$ ) the second-order contribution to the spin asymmetry energy $S\left(k_{f}\right)$ of nuclear matter can be calculated. One obtains the result:

$$
\begin{aligned}
S\left(k_{f}\right)^{(2 \mathrm{nd})=}= & \frac{M k_{f}^{4}}{60 \pi^{4}}\left\{t_{0}^{2}\left[\left(1+x_{0}^{2}\right)(1-2 \ln 2)+10 x_{0}\right]\right. \\
& +\frac{k_{f}^{2}}{42} t_{0} t_{1}\left[4\left(1+x_{0} x_{1}\right)(51-22 \ln 2)+7\left(x_{0}+x_{1}\right)(57-4 \ln 2)\right] \\
& +\frac{k_{f}^{4}}{63} t_{1}^{2}\left[\left(1+x_{1}^{2}\right)\left(\frac{1301}{6}-46 \ln 2\right)+x_{1}(607-48 \ln 2)\right] \\
& +\frac{k_{f}^{4}}{567} t_{2}^{2}\left[5\left(1+x_{2}^{2}\right)\left(\frac{655}{2}-78 \ln 2\right)+x_{2}(3187-624 \ln 2)\right] \\
& +\frac{k_{f}^{2}}{6} t_{0} t_{2}\left(2-x_{0}+x_{2}-2 x_{0} x_{2}\right)(4 \ln 2-7) \\
& +\frac{2 k_{f}^{4}}{63} t_{1} t_{2}\left(2-x_{1}+x_{2}-2 x_{1} x_{2}\right)(12 \ln 2-31) \\
& \left.+\frac{10 k_{f}^{4}}{189} W_{0}^{2}(227-96 \ln 2)\right\},
\end{aligned}
$$

which also includes $s p$-interference terms proportional to $t_{0} t_{2}$ and $t_{1} t_{2}$. When treating the spin-orbit interaction at second order, the spin-trace over three polarized medium-insertions eq.(7) singles out the third component of the vector $\vec{b}=\vec{q}_{1} \times \vec{q}_{2}$, but by spherical symmetry of the integration region one can make replacement $b_{3}^{2} \rightarrow \vec{b}^{2} / 3$. A good check of the intricate second-order calculation of asymmetry energies is provided by the relation:

$$
S\left(k_{f}\right)^{(2 \mathrm{nd})}=A\left(k_{f}\right)_{x_{0,1} \rightarrow-x_{0,1}}^{(2 \mathrm{nd})}-\frac{M k_{f}^{8}}{630 \pi^{4}} W_{0}^{2}(43+24 \ln 2),
$$


whose first part follows by working with the equivalent interaction formulated in terms of the isospin-exchange operator $P_{\tau}=\left(1+\vec{\tau}_{1} \cdot \vec{\tau}_{2}\right) / 2$ instead of $P_{\sigma}=\left(1+\vec{\sigma}_{1} \cdot \vec{\sigma}_{2}\right) / 2$. Obviously, the spin-orbit interaction produces different responses to asymmetries in isospin-space and spin-space.

Finally, by reweighting the Hartree and Fock diagrams (with factors $1 / 2$ and 1 ) one obtains the following second-order contribution to the spin asymmetry energy of pure neutron matter:

$$
\begin{aligned}
S_{n}\left(k_{n}\right)^{(2 \mathrm{nd})}= & \frac{M k_{n}^{4}}{90 \pi^{4}}\left\{-t_{0}^{2}\left(1-x_{0}\right)^{2}(2+\ln 2)-\frac{5 k_{n}^{2}}{28} t_{0} t_{1}\left(1-x_{0}\right)\left(1-x_{1}\right)(13+4 \ln 2)\right. \\
& -\frac{k_{n}^{4}}{189} t_{1}^{2}\left(1-x_{1}\right)^{2}(130+33 \ln 2)+\frac{k_{n}^{4}}{126} t_{2}^{2}\left(1+x_{2}\right)^{2}(359-78 \ln 2) \\
& +\frac{k_{n}^{2}}{4} t_{0} t_{2}\left(1-x_{0}\right)\left(1+x_{2}\right)(4 \ln 2-7)+\frac{k_{n}^{4}}{21} t_{1} t_{2}\left(1-x_{1}\right)\left(1+x_{2}\right)(12 \ln 2-31) \\
& \left.+\frac{10 k_{n}^{4}}{189} W_{0}^{2}(227-96 \ln 2)\right\} .
\end{aligned}
$$

This expression includes $s p$-mixing terms and it depends on the expected parameter combinations.

\section{Effective nucleon mass and Fermi-liquid parameters}

In this section, we study the effective nucleon mass and the isotropic Fermi-liquid parameters. Through interactions with the dense nuclear medium, nucleons acquire a momentum- and densitydependent self-energy and thereby turn into quasi-particles. Long-lived quasi-particle excitations exist only in the vicinity the Fermi surface $|\vec{p}|=k_{f}$ and they are (kinematically) characterized by an effective mass. The ratio between the free nucleon mass $M$ and the density-dependent in-medium nucleon mass $M^{*}\left(k_{f}\right)$ can be written in the form:

$$
\frac{M}{M^{*}\left(k_{f}\right)}=1-\frac{k_{f}^{2}}{2 M^{2}}+R\left(k_{f}\right),
$$

where the second term $-k_{f}^{2} / 2 M^{2}$ stems from the relativistic correction $-p^{4} / 8 M^{3}$ to the kinetic energy. The effects of interactions are represented by the (dimensionless) quantity $R\left(k_{f}\right)=$ $-2 M k_{f} f_{1}\left(k_{f}\right) / 3 \pi^{2}$ with $f_{1}\left(k_{f}\right)$ the spin- and isospin-independent $p$-wave Landau parameter [8]. The first order contribution from the Skyrme interaction $V_{\mathrm{Sk}}$ to the quantity $R\left(k_{f}\right)$ reads:

$$
R\left(k_{f}\right)^{(1 \mathrm{st})}=\frac{M k_{f}^{3}}{12 \pi^{2}}\left[3 t_{1}+t_{2}\left(5+4 x_{2}\right)\right] .
$$

In order to determine the corresponding second-order contribution, one has to evaluate the five direct and crossed one-loop diagrams (and their mirror-reflected partners) for forward scattering of two nucleons on the Fermi surface $\left|\vec{p}_{1}\right|=\left|\vec{p}_{2}\right|=k_{f}$, shown in Fig. 2 . The $p$-wave projection is performed by angular averaging with a weighting factor $3 \hat{p}_{1} \cdot \hat{p}_{2}$. After a somewhat lengthy calculation one arrives at the following result:

$$
\begin{aligned}
R\left(k_{f}\right)^{(2 \mathrm{nd})=} & \frac{M^{2} k_{f}^{2}}{10 \pi^{4}}\left\{t_{0}^{2}\left(1+x_{0}^{2}\right)(1-7 \ln 2)+\frac{k_{f}^{2}}{14} t_{0} t_{1}\left(1+x_{0} x_{1}\right)(47-104 \ln 2)\right. \\
& +\frac{k_{f}^{4}}{378} t_{1}^{2}\left(1+x_{1}^{2}\right)(911-942 \ln 2)+\frac{k_{f}^{4}}{1134} t_{2}^{2}\left(5+8 x_{2}+5 x_{2}^{2}\right)(313-426 \ln 2) \\
& \left.+\frac{k_{f}^{4}}{189} W_{0}^{2}(881-1032 \ln 2)\right\},
\end{aligned}
$$



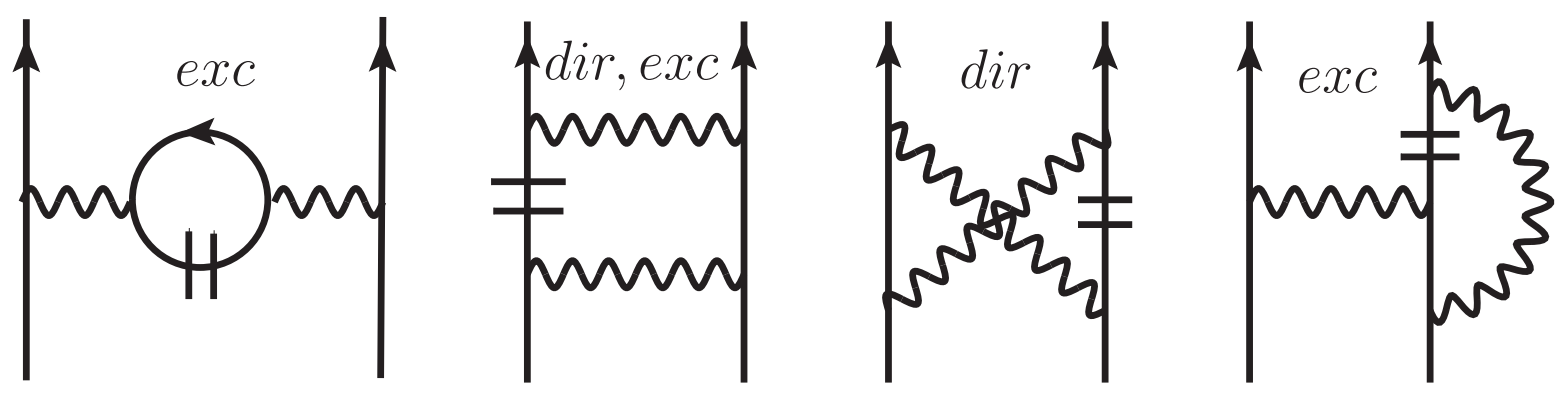

Figure 2: Direct and exchange diagrams representing the quasi-particle interaction at second order. Exchange diagrams need to be multiplied by the negative exchange operator $-P_{\tau} P_{\sigma}$.

which interestingly depends on the same combinations of parameters $t_{0}^{2}\left(1+x_{0}^{2}\right), t_{0} t_{1}\left(1+x_{0} x_{1}\right)$, $t_{1}^{2}\left(1+x_{1}^{2}\right), t_{2}^{2}\left(5+8 x_{2}+5 x_{2}^{2}\right)$ and $W_{0}^{2}$ as the second-order energy per particle $\bar{E}\left(k_{f}\right)^{(2 \mathrm{nd})}$ written in eq. (16).

The isotropic (i.e. angle-averaged) quasi-particle interaction $\mathcal{F}_{0}\left(k_{f}\right)$ on the Fermi surface $\left|\vec{p}_{1,2}\right|=k_{f}$ can be decomposed into four density-dependent ( $s$-wave) Fermi-liquid parameters:

$$
\mathcal{F}_{0}\left(k_{f}\right)=f_{0}\left(k_{f}\right)+f_{0}^{\prime}\left(k_{f}\right) \vec{\tau}_{1} \cdot \vec{\tau}_{2}+g_{0}\left(k_{f}\right) \vec{\sigma}_{1} \cdot \vec{\sigma}_{2}+g_{0}^{\prime}\left(k_{f}\right) \vec{\tau}_{1} \cdot \vec{\tau}_{2} \vec{\sigma}_{1} \cdot \vec{\sigma}_{2}
$$

The importance of the isotropic Fermi-liquid parameters $f_{0}\left(k_{f}\right), f_{0}^{\prime}\left(k_{f}\right)$ and $g_{0}\left(k_{f}\right)$ stems from the fact that these obey exact relations to specific nuclear matter properties. The Landau relation [8] to the nuclear matter incompressibility $K\left(k_{f}\right)$ reads:

$$
K\left(k_{f}\right)=k_{f}^{2} \frac{\partial^{2} \bar{E}\left(k_{f}\right)}{\partial k_{f}^{2}}+4 k_{f} \frac{\partial \bar{E}\left(k_{f}\right)}{\partial k_{f}}=\frac{3 k_{f}^{2}}{M}\left\{1-\frac{k_{f}^{2}}{2 M^{2}}+R\left(k_{f}\right)+\frac{2 M k_{f}}{\pi^{2}} f_{0}\left(k_{f}\right)\right\}
$$

where the expression on the right hand side is built up in the order: free nucleons with relativistic corrections, non-interacting quasi-nucleons, and finally interacting quasi-nucleons. The spin- and isospin-independent Fermi-liquid parameter $f_{0}\left(k_{f}\right)$ receives a first-order contribution of the form:

$$
f_{0}\left(k_{f}\right)^{(1 \mathrm{st})}=\frac{3 t_{0}}{4}+\frac{k_{f}^{2}}{8}\left[3 t_{1}+t_{2}\left(5+4 x_{2}\right)\right]
$$

and the second-order contribution derived from the one-loop scattering diagrams in Fig. 2 reads:

$$
\begin{aligned}
f_{0}\left(k_{f}\right)^{(2 \mathrm{nd})}= & \frac{M k_{f}}{4 \pi^{2}}\left\{t_{0}^{2}\left(1+x_{0}^{2}\right)(2+\ln 2)+\frac{k_{f}^{2}}{10} t_{0} t_{1}\left(1+x_{0} x_{1}\right)(41+8 \ln 2)\right. \\
& +\frac{k_{f}^{4}}{15} t_{1}^{2}\left(1+x_{1}^{2}\right)(32+3 \ln 2)+\frac{k_{f}^{4}}{63} t_{2}^{2}\left(5+8 x_{2}+5 x_{2}^{2}\right)(8+3 \ln 2) \\
& \left.+\frac{k_{f}^{4}}{105} W_{0}^{2}(463+24 \ln 2)\right\} .
\end{aligned}
$$

Note that in $f_{0}\left(k_{f}\right)^{(2 n d)}$ the same combinations of Skyrme parameters appear as in eqs. $(16,24)$. The validity of the Landau relation eq. (26) between $\bar{E}\left(k_{f}\right), R\left(k_{f}\right)$ and $f_{0}\left(k_{f}\right)$ is immediately verified at first and second order and thus serves as an important consistency check of our calculation.

There exists a Landau relation [8] for the isospin asymmetry energy $A\left(k_{f}\right)$ of nuclear matter:

$$
A\left(k_{f}\right)=\frac{k_{f}^{2}}{6 M}\left\{1-\frac{k_{f}^{2}}{2 M^{2}}+R\left(k_{f}\right)+\frac{2 M k_{f}}{\pi^{2}} f_{0}^{\prime}\left(k_{f}\right)\right\}
$$


which reflects again the composition: free nucleons with relativistic corrections, non-interacting quasi-nucleons, and interacting quasi-nucleons. The isospin-dependent Fermi-liquid parameter $f_{0}^{\prime}\left(k_{f}\right)$ receives a first-order contribution of the form:

$$
f_{0}^{\prime}\left(k_{f}\right)^{(1 \mathrm{st})}=-\frac{t_{0}}{4}\left(1+2 x_{0}\right)+\frac{k_{f}^{2}}{8}\left[t_{2}\left(1+2 x_{2}\right)-t_{1}\left(1+2 x_{1}\right)\right]
$$

and the second-order contribution derived from the one-loop scattering diagrams in Fig. 2 reads:

$$
\begin{aligned}
f_{0}^{\prime}\left(k_{f}\right)^{(2 \mathrm{nd})}= & \frac{M k_{f}}{4 \pi^{2}}\left\{t_{0}^{2}\left[\left(1+x_{0}^{2}\right) \ln 2-2 x_{0}\right]\right. \\
& +\frac{k_{f}^{2}}{30} t_{0} t_{1}\left[\left(1+x_{0} x_{1}\right)(9+32 \ln 2)+\left(x_{0}+x_{1}\right)(4 \ln 2-57)\right] \\
& +\frac{k_{f}^{4}}{315} t_{1}^{2}\left[\left(1+x_{1}^{2}\right)(65+111 \ln 2)+x_{1}(48 \ln 2-607)\right] \\
& +\frac{k_{f}^{4}}{63} t_{2}^{2}\left[\left(1+x_{2}^{2}\right)(19+15 \ln 2)+x_{2}(43+24 \ln 2)\right] \\
& +\frac{k_{f}^{2}}{30} t_{0} t_{2}\left(2+x_{0}+x_{2}+2 x_{0} x_{2}\right)(4 \ln 2-7) \\
& +\frac{2 k_{f}^{4}}{315} t_{1} t_{2}\left(2+x_{1}+x_{2}+2 x_{1} x_{2}\right)(12 \ln 2-31) \\
& \left.+\frac{k_{f}^{4}}{45} W_{0}^{2}(103+24 \ln 2)\right\} .
\end{aligned}
$$

It is important to note that the Landau relation eq. (29) between $A\left(k_{f}\right), R\left(k_{f}\right)$ and $f_{0}^{\prime}\left(k_{f}\right)$ is valid at first and second order.

In an analogous way the spin asymmetry energy $S\left(k_{f}\right)$ of nuclear matter is related to spin-spin part of the (isotropic) quasi-particle interaction:

$$
S\left(k_{f}\right)=\frac{k_{f}^{2}}{6 M}\left\{1-\frac{k_{f}^{2}}{2 M^{2}}+R\left(k_{f}\right)+\frac{2 M k_{f}}{\pi^{2}} g_{0}\left(k_{f}\right)\right\} .
$$

The spin-dependent Fermi-liquid parameter $g_{0}\left(k_{f}\right)$ receives from $V_{\mathrm{Sk}}$ the first-order contribution:

$$
g_{0}\left(k_{f}\right)^{(1 \mathrm{st})}=\frac{t_{0}}{4}\left(2 x_{0}-1\right)+\frac{k_{f}^{2}}{8}\left[t_{1}\left(2 x_{1}-1\right)+t_{2}\left(1+2 x_{2}\right)\right]=f_{0}^{\prime}\left(k_{f}\right)_{x_{0,1} \rightarrow-x_{0,1}}^{(1 \mathrm{st})}
$$

and the second-order contribution derived from the one-loop scattering diagrams in Fig. 2 reads:

$$
g_{0}\left(k_{f}\right)^{(2 \mathrm{nd})}=f_{0}^{\prime}\left(k_{f}\right)_{x_{0,1} \rightarrow-x_{0}, 1}^{(2 \mathrm{nd})}-\frac{M k_{f}^{5}}{210 \pi^{2}} W_{0}^{2}(43+24 \ln 2),
$$

with $f_{0}^{\prime}\left(k_{f}\right)^{(2 n d)}$ written in eq.(31). The Landau relation eq.(32) between $S\left(k_{f}\right), R\left(k_{f}\right)$ and $g_{0}\left(k_{f}\right)$ is just as well satisfied at first and second order.

Finally, there is the isovector spin-spin Fermi-liquid parameter $g_{0}^{\prime}\left(k_{f}\right)$. The corresponding contributions from the Skyrme interaction $V_{\mathrm{Sk}}$ at first and second order read:

$$
g_{0}^{\prime}\left(k_{f}\right)^{(1 \mathrm{st})}=-\frac{t_{0}}{4}+\frac{k_{f}^{2}}{8}\left(t_{2}-t_{1}\right)
$$




$$
\begin{aligned}
g_{0}^{\prime}\left(k_{f}\right)^{(2 \mathrm{nd})}= & \frac{M k_{f}}{12 \pi^{2}}\left\{t_{0}^{2}\left[3 \ln 2-x_{0}^{2}(2+\ln 2)\right]+\frac{k_{f}^{2}}{10} t_{0} t_{1}\left[9+32 \ln 2-x_{0} x_{1}(41+8 \ln 2)\right]\right. \\
& +\frac{k_{f}^{4}}{105} t_{1}^{2}\left[65+111 \ln 2-7 x_{1}^{2}(32+3 \ln 2)\right] \\
& +\frac{k_{f}^{4}}{21} t_{2}^{2}\left[19+22 x_{2}+8 x_{2}^{2}+3\left(5+8 x_{2}+x_{2}^{2}\right) \ln 2\right]+\frac{k_{f}^{2}}{5} t_{0} t_{2}\left(1+x_{2}\right)(4 \ln 2-7) \\
& \left.+\frac{4 k_{f}^{4}}{105} t_{1} t_{2}\left(1+x_{2}\right)(12 \ln 2-31)+\frac{k_{f}^{4}}{45} W_{0}^{2}(103+24 \ln 2)\right\} .
\end{aligned}
$$

Note that $s p$-mixing terms are present in the three spin- or isospin-dependent Fermi-liquid parameters.

Additional checks of our calculation are provided by the Fermi-liquid parameters $f_{n 0}\left(k_{n}\right)$ and $g_{n 0}\left(k_{n}\right)$ of neutrons in pure neutron matter. In order to obtain these from the computation of the isotropic quasi-particle interaction $\mathcal{F}_{0}\left(k_{f}\right)$ in isospin-symmetric nuclear matter, one merely has to set $P_{\tau}=1$, replace $k_{f} \rightarrow k_{n}$, and multiply the left diagram in Fig. 2 (with a closed nucleon ring) by a factor $1 / 2$. The $p$-wave Landau parameter $f_{n 1}\left(k_{n}\right)$ enters the effective neutron mass ratio $R_{n}\left(k_{n}\right)$ with a factor $-M k_{n} / 3 \pi^{2}$. For the sake of completeness we list the relevant expressions for the neutron quasi-particle properties at first order:

$$
\begin{gathered}
R_{n}\left(k_{n}\right)^{(1 \mathrm{st})}=\frac{M k_{n}^{3}}{12 \pi^{2}}\left[t_{1}\left(1-x_{1}\right)+3 t_{2}\left(1+x_{2}\right)\right] \\
f_{n 0}\left(k_{n}\right)^{(1 \mathrm{st})}=\frac{t_{0}}{2}\left(1-x_{0}\right)+\frac{k_{n}^{2}}{4}\left[t_{1}\left(1-x_{1}\right)+3 t_{2}\left(1+x_{2}\right)\right], \\
g_{n 0}\left(k_{n}\right)^{(1 \mathrm{st})}=\frac{t_{0}}{2}\left(x_{0}-1\right)+\frac{k_{n}^{2}}{4}\left[t_{1}\left(x_{1}-1\right)+t_{2}\left(1+x_{2}\right)\right],
\end{gathered}
$$

and at second order:

$$
\begin{aligned}
& R_{n}\left(k_{n}\right)^{(2 \mathrm{nd})}= \frac{M^{2} k_{n}^{2}}{30 \pi^{4}}\left\{t_{0}^{2}\left(1-x_{0}\right)^{2}(1-7 \ln 2)+\frac{k_{n}^{2}}{14} t_{0} t_{1}\left(1-x_{0}\right)\left(1-x_{1}\right)(47-104 \ln 2)\right. \\
&+\frac{k_{n}^{4}}{378} t_{1}^{2}\left(1-x_{1}\right)^{2}(911-942 \ln 2)+\frac{k_{n}^{4}}{126} t_{2}^{2}\left(1+x_{2}\right)^{2}(313-426 \ln 2) \\
&\left.+\frac{2 k_{n}^{4}}{189} W_{0}^{2}(881-1032 \ln 2)\right\} \\
& f_{n 0}\left(k_{n}\right)^{(2 \mathrm{nd})}= \frac{M k_{n}}{6 \pi^{2}}\left\{t_{0}^{2}\left(1-x_{0}\right)^{2}(2+\ln 2)+\frac{k_{n}^{2}}{10} t_{0} t_{1}\left(1-x_{0}\right)\left(1-x_{1}\right)(41+8 \ln 2)\right. \\
&+\frac{k_{n}^{4}}{15} t_{1}^{2}\left(1-x_{1}\right)^{2}(32+3 \ln 2)+\frac{k_{n}^{4}}{7} t_{2}^{2}\left(1+x_{2}\right)^{2}(8+3 \ln 2) \\
&\left.+\frac{2 k_{n}^{4}}{105} W_{0}^{2}(463+24 \ln 2)\right\} \cdot \\
& g_{n 0}\left(k_{n}\right)^{(2 \mathrm{nd})}=\frac{M k_{n}}{6 \pi^{2}}\left\{t_{0}^{2}\left(1-x_{0}\right)^{2}(\ln 2-1)+\frac{2 k_{n}^{2}}{5} t_{0} t_{1}\left(1-x_{0}\right)\left(1-x_{1}\right)(3 \ln 2-4)\right. \\
&+\frac{k_{n}^{4}}{70} t_{1}^{2}\left(1-x_{1}\right)^{2}(30 \ln 2-53)+\frac{3 k_{n}^{4}}{14} t_{2}^{2}\left(1+x_{2}\right)^{2}(3+2 \ln 2) \\
&+\frac{k_{n}^{2}}{10} t_{0} t_{2}\left(1-x_{0}\right)\left(1+x_{2}\right)(4 \ln 2-7)+\frac{2 k_{n}^{4}}{105} t_{1} t_{2}\left(1-x_{1}\right)\left(1+x_{2}\right)(12 \ln 2-31) \\
&\left.+\frac{2 k_{n}^{4}}{315} W_{0}^{2}(463+24 \ln 2)\right\} .
\end{aligned}
$$


The pertinent Landau relations to the incompressibility $K_{n}\left(k_{n}\right)$ and spin asymmetry energy $S_{n}\left(k_{n}\right)$ of pure neutron matter (analogous to eqs. $(26,32)$ but with a prefactor $M k_{n} / \pi^{2}$ at the last summand) are satisfied at first and second order. The quasi-particle interaction of neutrons in pure neutron matter, taking into account also the non-central tensor and cross-vector terms, has been calculated in ref. [15] on the basis of chiral two- and three-nucleon potentials in many-body perturbation theory up to second order.

\section{Tensor interactions}

The Skyrme interaction $V_{\mathrm{Sk}}$ in eq.(1) depends on seven parameters. This are two less than the nine low-energy constants $C_{S, T}$ and $C_{j}, j=1, \ldots, 7$ appearing in general NN contact-potential of chiral effective field theory [10, 11, 12] up to order $p^{2}$. A complete matching a achieved by supplementing to the Skyrme interaction $V_{\mathrm{Sk}}$ two additional tensor terms of the Galilei- and time-reversal-invariant form:

$$
\begin{aligned}
V_{\text {ten }}= & t_{4}\left[3 \vec{\sigma}_{1} \cdot \vec{q}_{\text {out }} \vec{\sigma}_{2} \cdot \vec{q}_{\text {out }}+3 \vec{\sigma}_{1} \cdot \vec{q}_{\text {in }} \vec{\sigma}_{2} \cdot \vec{q}_{\text {in }}-\vec{\sigma}_{1} \cdot \vec{\sigma}_{2}\left(\vec{q}_{\text {out }}^{2}+\vec{q}_{\text {in }}^{2}\right)\right] \\
& +t_{5}\left[3 \vec{\sigma}_{1} \cdot \vec{q}_{\text {out }} \vec{\sigma}_{2} \cdot \vec{q}_{\text {in }}+3 \vec{\sigma}_{1} \cdot \vec{q}_{\text {in }} \vec{\sigma}_{2} \cdot \vec{q}_{\text {out }}-2 \vec{\sigma}_{1} \cdot \vec{\sigma}_{2} \vec{q}_{\text {out }} \cdot \vec{q}_{\text {in }}\right] .
\end{aligned}
$$

Note that for both terms in $V_{\text {ten }}$ the spin-matrices $\sigma_{1}^{i} \sigma_{2}^{j}$ are contracted with symmetric and traceless tensors depending on the external momentum-differences $\vec{q}_{\text {in }}$ and $\vec{q}_{\text {out }}$. Because of these properties the first-order contribution from $V_{\text {ten }}$ as well as all its interference terms with $V_{\text {Sk }}$ vanish, regarding energies per particle and the central quasi-particle interaction in nuclear matter.

Using dimensional regularization (as discussed in the beginning of section 3) and evaluating the second-order Hartree and Fock diagrams in Fig. 1 with the tensorial contact-interaction $V_{\text {ten }}$, one obtains the following contributions to the energy per particle of isospin-symmetric nuclear matter:

$$
\bar{E}\left(k_{f}\right)^{(\text {ten })}=\frac{M k_{f}^{8}}{15 \pi^{4}}\left\{\frac{t_{4}^{2}}{231}(1525-129 \ln 2)+t_{5}^{2}(19-3 \ln 2)\right\},
$$

and to the energy per particle of pure neutron matter:

$$
\bar{E}_{n}\left(k_{n}\right)^{(\text {ten })}=\frac{2 M k_{n}^{8}}{45 \pi^{4}} t_{5}^{2}(19-3 \ln 2) .
$$

The asymmetric medium-insertions introduced in eqs. $(4,7)$ lead to the following second-order contributions from $V_{\text {ten }}$ to the isospin asymmetry energy of nuclear matter:

$$
A\left(k_{f}\right)^{(\text {ten })}=\frac{M k_{f}^{8}}{945 \pi^{4}}\left\{t_{5}^{2}(3719-708 \ln 2)-t_{4}^{2}\left(100 \ln 2+\frac{317}{3}\right)+24 t_{4} t_{5}(12 \ln 2-31)\right\},
$$

and to the spin asymmetry energy of nuclear matter:

$$
S\left(k_{f}\right)^{(\text {ten })}=\frac{M k_{f}^{8}}{54 \pi^{4}}\left\{\frac{11 t_{4}^{2}}{15}(13-48 \ln 2)+\frac{t_{5}^{2}}{7}(395-1104 \ln 2)\right\} .
$$

Interestingly, the $t_{4} t_{5}$-interference term is absent in $\bar{E}\left(k_{f}\right)^{(\text {ten })}$ and $S\left(k_{f}\right)^{(\text {ten })}$. The actual calculation of the second-order Hartree diagram produces in both cases an integrand $H=t_{4} t_{5}\left(\left|\vec{q}_{1}\right|^{4}-\left|\vec{q}_{2}\right|^{4}\right)$ for the triple Fermi-sphere integral in eq.(15), but due to the symmetry $\vec{q}_{1} \leftrightarrow \vec{q}_{2}$ of the other ingredients it integrates to zero. By reweighting the Hartree diagram with a factor $1 / 2$, the second-order contribution from $V_{\text {ten }}$ to the spin asymmetry energy of pure neutron matter follows as:

$$
S_{n}\left(k_{n}\right)^{(\text {ten })}=\frac{M k_{n}^{8}}{567 \pi^{4}} t_{5}^{2}(395-1104 \ln 2)
$$




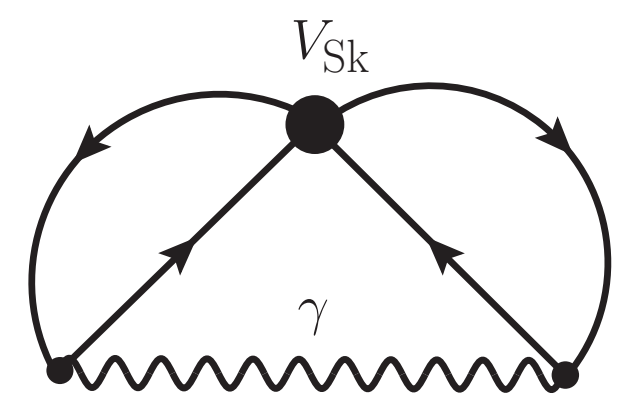

Figure 3: Iteration of the zero-range Skyrme interaction with one-photon exchange.

One observes that the neutron matter quantities written in eqs. $(39,42)$ depend only on the parameter $t_{5}$. This feature is explained by the fact the first tensor term in eq.(37) proportional to $t_{4}$ is responsible for the mixing between the ${ }^{3} S_{1}$-state and ${ }^{3} D_{1}$-state with total isospin $I=0$.

We continue with presenting the second-order contributions from $V_{\text {ten }}$ to the effective nucleon mass ratio:

$$
R\left(k_{f}\right)^{(\mathrm{ten})}=\frac{M^{2} k_{f}^{6}}{126 \pi^{4}}\left\{\frac{t_{4}^{2}}{3}(929-888 \ln 2)+\frac{t_{5}^{2}}{5}(5147-6504 \ln 2)\right\},
$$

and to the isotropic Fermi-liquid parameters:

$$
\begin{gathered}
f_{0}\left(k_{f}\right)^{(\text {ten })}=\frac{M k_{f}^{5}}{140 \pi^{2}}\left\{t_{4}^{2}\left(\frac{2195}{3}+88 \ln 2\right)+t_{5}^{2}(2029+312 \ln 2)\right\}, \\
f_{0}^{\prime}\left(k_{f}\right)^{(\text {ten })}=\frac{M k_{f}^{5}}{140 \pi^{2}}\left\{3 t_{4}^{2}(40 \ln 2-73)+\frac{32}{3} t_{4} t_{5}(12 \ln 2-31)+t_{5}^{2}(1081+408 \ln 2)\right\}, \\
g_{0}\left(k_{f}\right)^{(\text {ten })}=-\frac{M k_{f}^{5}}{20 \pi^{2}}\left\{\frac{t_{4}^{2}}{63}(881+984 \ln 2)+t_{5}^{2}(19+72 \ln 2)\right\}, \\
g_{0}^{\prime}\left(k_{f}\right)^{(\text {ten })}=\frac{M k_{f}^{5}}{140 \pi^{2}}\left\{t_{4}^{2}(40 \ln 2-73)+\frac{32}{9} t_{4} t_{5}(31-12 \ln 2)-\frac{t_{5}^{2}}{9}(1613+2424 \ln 2)\right\} .
\end{gathered}
$$

Note that $t_{4} t_{5}$-interference terms are present only in the isovectorial quantities $f_{0}^{\prime}\left(k_{f}\right)$ and $g_{0}^{\prime}\left(k_{f}\right)$. The simultaneous validity of the three Landau relations in eqs. $(26,29,32)$ provides an excellent check of our second-order calculation with the zero-range tensor interaction $V_{\text {ten }}$. The secondorder contributions from $V_{\text {ten }}$ to the neutron quasi-particle properties are obviously proportional to $t_{5}^{2}$ and they read:

$$
\begin{gathered}
R_{n}\left(k_{n}\right)^{(\text {ten })}=\frac{M^{2} k_{n}^{6}}{945 \pi^{4}} t_{5}^{2}(5147-6504 \ln 2) \\
f_{n 0}\left(k_{n}\right)^{(\text {ten })}=\frac{M k_{n}^{5}}{105 \pi^{2}} t_{5}^{2}(2029+312 \ln 2), \quad g_{n 0}\left(k_{n}\right)^{(\text {ten })}=-\frac{M k_{n}^{5}}{15 \pi^{2}} t_{5}^{2}(19+72 \ln 2) .
\end{gathered}
$$

\section{Iteration of $V_{\mathrm{Sk}}$ with one-photon exchange}

The Skyrme interaction $V_{\mathrm{Sk}}$ in eq.(1) can be viewed as a suitable phenomenological parameterization of the strong nuclear force. In nuclear matter (or finite nuclei) it affects protons and neutrons in the same way. This isospin-degeneracy gets lifted by taking into account the Coulomb interaction between the protons. A further charge-symmetry breaking contribution is provided by iterating the (strong) Skyrme interaction $V_{\mathrm{Sk}}$ with the (electromagnetic) one-photon exchange, as depicted by the three-loop diagram in Fig. 3. In the case at hand the iterated diagram with two 
medium-insertions does not vanish in dimensional regularization due to the presence of a photonpropagator $\left[m_{\gamma}^{2}+(\vec{l} \pm \vec{q})^{2}\right]^{-1}$, with $m_{\gamma}$ an infinitesimal photon mass. The limit $m_{\gamma} \rightarrow 0$ has to be taken after performing the $d^{3} l$-loop integral, which results in: $\arctan \left(2|\vec{q}| / m_{\gamma}\right) \rightarrow \pi / 2$. After combination with the Pauli-blocking corrections (supplied by the iterated diagram with three medium-insertions) one obtains the following contribution to the energy per proton:

$$
\begin{aligned}
\bar{E}_{p}\left(k_{p}\right)= & \frac{\alpha M k_{p}^{2}}{10 \pi^{3}}\left\{t_{0}\left(1-x_{0}\right)\left(1-\frac{\pi^{2}}{3}-2 \ln 2\right)+\frac{3 k_{p}^{2}}{14}\left[t_{1}\left(1-x_{1}\right)\left(\frac{31}{4}-\frac{\pi^{2}}{3}-3 \ln 2\right)\right.\right. \\
& \left.\left.+t_{2}\left(1+x_{2}\right)\left(\frac{5}{4}-\pi^{2}-5 \ln 2\right)+\frac{2 \mu_{p}^{2}}{3 M^{2}} t_{0}\left(1-x_{0}\right)(11-2 \ln 2)\right]\right\},
\end{aligned}
$$

where $\alpha=e^{2} / 4 \pi=1 / 137.036$ denotes the electromagnetic fine-structure constant and the proton Fermi momentum $k_{p}$ is related to the proton density by $\rho_{p}=k_{p}^{3} / 3 \pi^{2}$. Remarkably, the result for $\bar{E}_{p}\left(k_{p}\right)$ is infrared-finite, since an infrared regulator term $\ln \left(k_{p} / m_{\gamma}\right)$ drops out in the end by angular integration: $\int_{-1}^{1} d y[f(y)-f(-y)]=0$. The last term in eq.(56) comes from iterating the $s$-wave contact part $t_{0}\left(1-x_{0}\right)$ with the magnetic interaction $\sim \vec{\sigma} \times \overrightarrow{q_{j}}$, where $\mu_{p}=2.79$ denotes the proton magnetic moment in units of the nuclear magneton $e / 2 M$. For the sake of completeness, we give also the contribution from the iterated diagram in Fig. 3 to the spin asymmetry energy of protons:

$$
\begin{aligned}
S_{p}\left(k_{p}\right)= & \frac{\alpha M k_{p}^{2}}{18 \pi^{3}}\left\{t_{0}\left(1-x_{0}\right)\left(\frac{2 \pi^{2}}{3}+1-2 \ln 2\right)+\frac{k_{p}^{2}}{5}\left[t_{1}\left(1-x_{1}\right)\left(\frac{2 \pi^{2}}{3}-\frac{47}{4}+\ln 2\right)\right.\right. \\
& \left.\left.-t_{2}\left(1+x_{2}\right)\left(\frac{4 \pi^{2}}{3}+\frac{5}{4}+5 \ln 2\right)+\frac{4 \mu_{p}^{2}}{M^{2}} t_{0}\left(x_{0}-1\right)(2+\ln 2)\right]\right\} .
\end{aligned}
$$

As an aside we note that the one-photon exchange with the magnetic coupling gives also (small) first-order Fock contributions of the form: $\bar{E}_{p}\left(k_{p}\right)=-3 S_{p}\left(k_{p}\right)=\alpha \mu_{p}^{2} k_{p}^{3} /\left(6 \pi M^{2}\right)$.

The charge-symmetry breaking contribution $\rho_{p} \bar{E}_{p}\left(k_{p}\right)$ to the energy density of protons in nuclei could play a role in resolving the Nolen-Schiffer anomaly [9, 16]. This anomaly refers to the observation that the binding energy differences of mirror nuclei cannot be explained by the Coulomb interaction of protons alone, using realistic nuclear wave functions. In a systematic study by Brown et al. [17] it was found that the anomaly can be resolved by either dropping the attractive Coulomb Fock-term $\bar{E}_{p}\left(k_{p}\right)=-3 \alpha k_{p} / 4 \pi$ or by introducing a charge-symmetry breaking delta-force. The first term in eq.(56) proportional to $k_{p}^{2}$ has been investigated in ref. [18] with respect to its capability of compensating the attractive Coulomb Fock-term. Such a study should be extended to the iteration of the complete Skyrme interaction $V_{\mathrm{Sk}}$ with the one-photon exchange. Actually, the novel charge-symmetry breaking contribution $\bar{E}_{p}\left(k_{p}\right)$ written in eq.(56) should be included routinely in Skyrme calculations of medium-mass and heavy nuclei.

\section{Provisional parameter fits}

In this section we perform some provisional parameter fits in order to learn about possible improvements through the inclusion of second-order contributions from $V_{\mathrm{Sk}}$. We restrict the discussion to the nuclear matter saturation curve in the density region $0<\rho<2 \rho_{0}=0.32 \mathrm{fm}^{-3}$. The equation of state provided by the phenomenological SLy5-interaction [19] is taken as a benchmark. Out of interest we perform first a fit to the energy per particle at first order: $\bar{E}\left(k_{f}\right)=\bar{E}\left(k_{f}\right)^{(\mathrm{kin})}-a_{3} k_{f}^{3}+a_{5} k_{f}^{5}$. The empirical nuclear matter saturation point $\left(k_{f 0}=263 \mathrm{MeV}, \bar{E}_{0}=-16 \mathrm{MeV}\right)$ fixes the two free parameters to the values: $a_{3}=30.81 \mathrm{MeVfm}^{3}, a_{5}=8.356 \mathrm{MeVfm}^{5}$, and the resulting saturation curve is shown by the full line in Fig. 4. It is considerably stiffer than the realistic equation of 


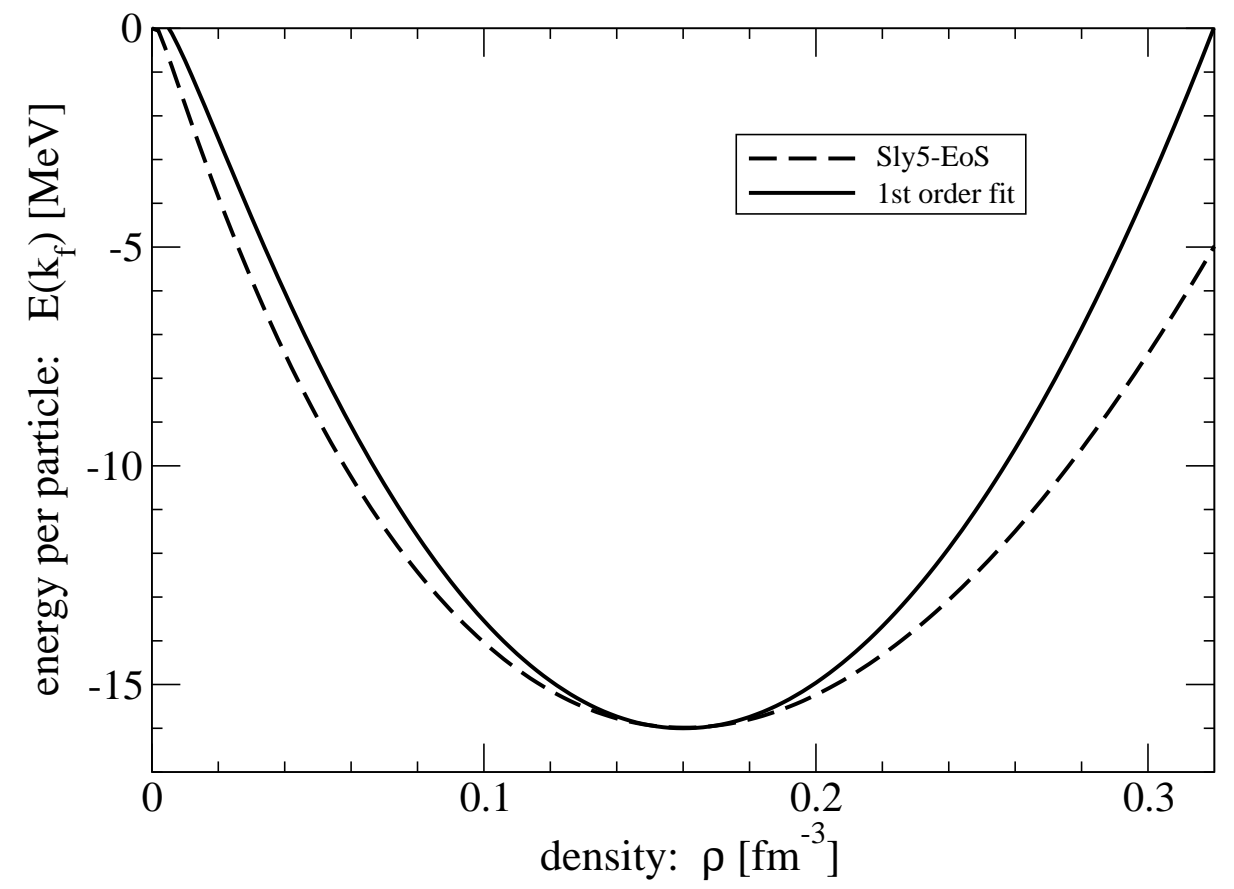

Figure 4: Saturation curve of isospin-symmetric nuclear matter. The dashed line stems from the SLy5-interaction [19]. The full curve is a first-order fit without the density-dependent term $\frac{1}{6} t_{3} \rho^{1 / 6}$.

state (represented by the dashed line in Fig. 4) as evidenced by the high value $K=307 \mathrm{MeV}$ of the nuclear matter incompressibility. While the shape of the full line in Fig. 4 can be considered as acceptable, there is serious problem behind it, namely the unrealistically low value of effective nucleon mass $M^{*}\left(k_{f 0}\right)=0.39 M$, which is completely determined by the parameter $a_{5}$. This (unwanted) rigid correlation at first order is usually avoided by introducing the density-dependent term $\frac{1}{6} t_{3}\left(1+x_{3} P_{\sigma}\right) \rho^{1 / 6}$.

Next, we include in the fit the complete second-order contribution $\bar{E}\left(k_{f}\right)^{(2 \mathrm{nd})}+\bar{E}\left(k_{f}\right)^{\text {(ten })}$ written in eqs. $(16,44)$. The nine parameters are determined by minimizing the squared deviations from the realistic nuclear matter saturation curve at about 40 equally-spaced points in the density region $0<\rho<2 \rho_{0}=0.32 \mathrm{fm}^{-3}$. The resulting fit values are: $t_{0}=-3.535 \mathrm{fm}^{2}, t_{1}=1.156 \mathrm{fm}^{4}$, $t_{2}=-3.942 \mathrm{fm}^{4}$, and the others very close to zero: $x_{0,1,2}=0, t_{4,5}=0, W_{0}=0$. An inspection of the full line in Fig. 5 reveals that a good reproduction of the realistic nuclear matter saturation curve cannot be obtained. The reason for this failure lies presumably in the too strong densitydependence $k_{f}^{8} \sim \rho^{8 / 3}$ of several second-order contributions. But interestingly with the above parameter values, the isospin asymmetry energy $A\left(k_{f 0}\right)=S\left(k_{f 0}\right)=33.0 \mathrm{MeV}$ comes out close to its empirical value. The previous free fit gave zero even for the spin-orbit parameter $W_{0}$. If one fixes it to its (large) empirical value $W_{0}=126 \mathrm{MeVfm}^{5}=0.638 \mathrm{fm}^{4}$ [19], the other parameters are fitted to: $t_{0}=-3.849 \mathrm{fm}^{2}, t_{1}=1.778 \mathrm{fm}^{4}, t_{2}=-3.341 \mathrm{fm}^{4}$, and $x_{0,1,2}=0, t_{4,5}=0$. The resulting equation of state is shown by the dashed-dotted line in Fig. 5 and one observes that its shape remains almost unchanged in comparison to the free fit. The values for the isospin asymmetry energy $A\left(k_{f 0}\right)=36.0 \mathrm{MeV}$ and the spin asymmetry energy $S\left(k_{f 0}\right)=32.3 \mathrm{MeV}$ have changed a bit.

From these findings one can conclude that the inclusion of the (ad hoc) density-dependent term $\frac{1}{6} t_{3}\left(1+x_{3} P_{\sigma}\right) \rho^{1 / 6}$ is indispensable for a realistic description of nuclear matter in the Skyrme approach. Formally, this extra term is taken into account by making the parameter substitutions: $t_{0} \rightarrow t_{0}+\frac{1}{6} t_{3} \rho^{1 / 6}$ and $t_{0} x_{0} \rightarrow t_{0} x_{0}+\frac{1}{6} t_{3} x_{3} \rho^{1 / 6}$, in all first- and second-order expressions. We have convinced ourselves that after this extension a good reproduction of the realistic nuclear matter saturation curve is possible at second order. This is in agreement with the findings of refs. [4, 5], 
where however incorrect expressions have been used for the second-order contributions. Certainly, the fitting of parameters of the Skyrme interaction $V_{\mathrm{Sk}}$, when treated to second order, should go beyond the mere saturation curve and include as constraints all possible available properties of nuclear matter. Such a comprehensive analysis goes beyond the scope of the present paper. But the analytical calculations of a large variety of nuclear matter properties to first and second order as performed in this work lay the foundation for such a future study.

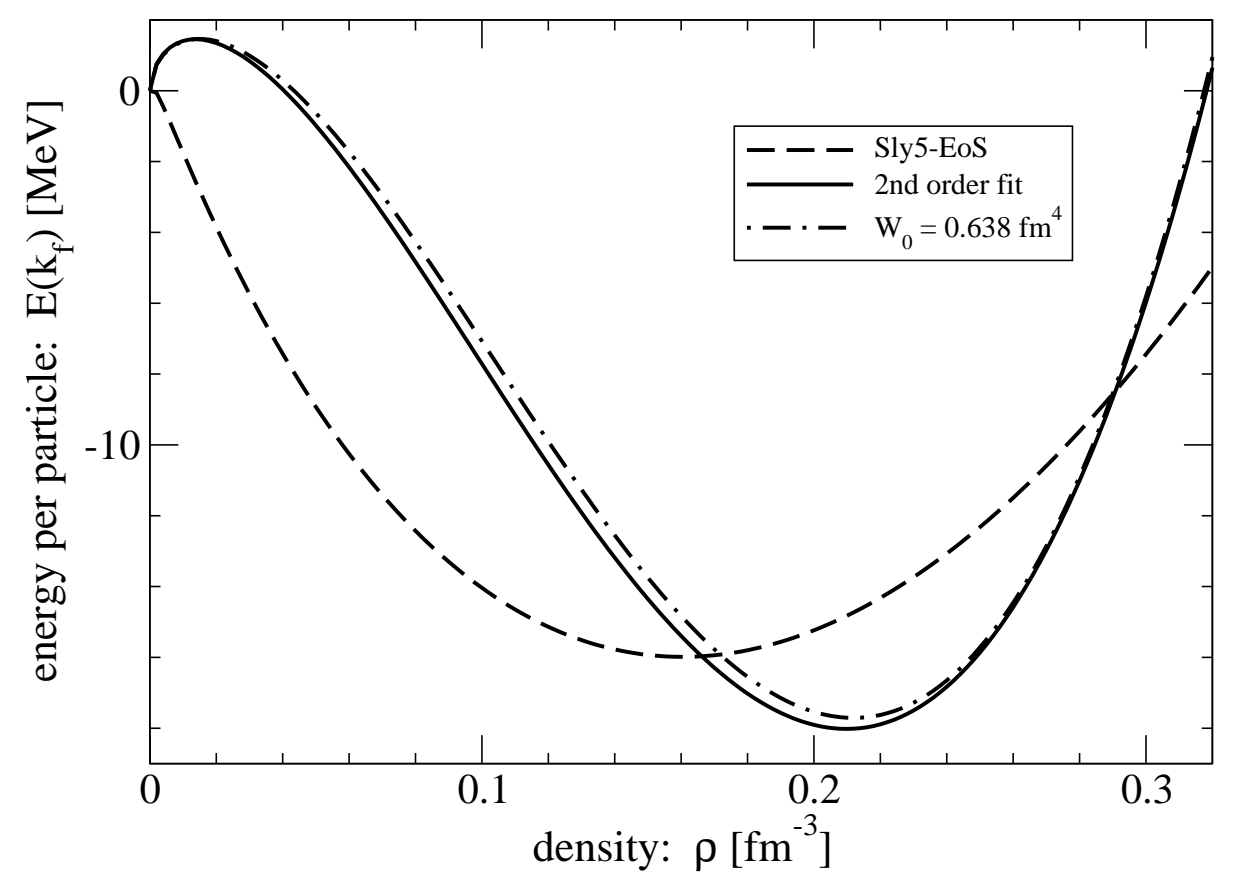

Figure 5: Fits to the nuclear matter saturation curve with inclusion of second-order contributions from the Skyrme interaction $V_{\mathrm{Sk}}$. The (ad hoc) density-dependent term $\frac{1}{6} t_{3} \rho^{1 / 6}$ is left out.

\section{Appendix A: In-medium loop featuring s-wave and p-wave mixing}

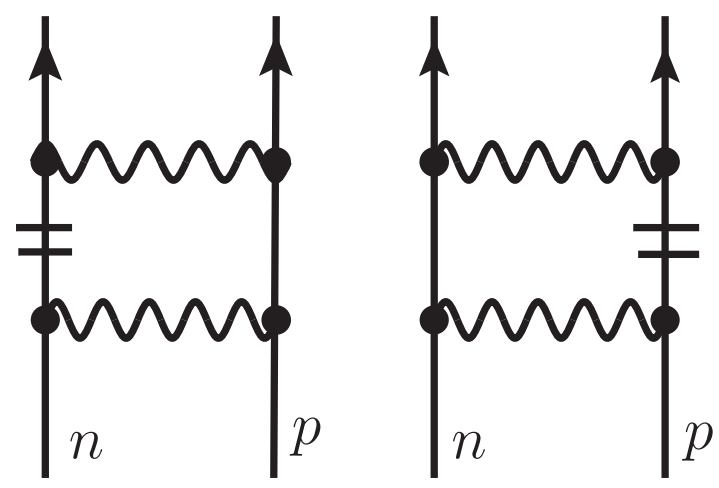

Figure 6: In-medium loop for an interacting proton-neutron pair. The short double-lines symbolize Pauli-blocking effects in the nuclear medium.

In this appendix we analyze under which conditions a mixing between $s$-wave and $p$-wave components of the two-body interaction (with different parity) can occur in a fermionic medium. 
The two diagrams in Fig. 6 represent the in-medium loop for an interacting proton-neutron pair in nuclear matter. The short double-line symbolizes the medium-insertion (i.e. the Pauli-blocking effect) for the neutron on the left side and for the proton on the right side. If the lower and upper contact-vertex are of $s$-wave type and $p$-wave type, respectively, the real part of the in-medium loop is given by a principal-value integral of the form:

$$
\begin{aligned}
& f \frac{d^{3} l}{(2 \pi)^{3}} \frac{\left(\overrightarrow{l^{2}}\right)^{j} \vec{l} \cdot \vec{q}}{\vec{l}^{2}-\vec{q}^{2}}\left\{\theta\left(k_{n}-|\vec{P}-\vec{l}|\right)+\theta\left(k_{p}-|\vec{P}+\vec{l}|\right)\right\} \\
& =f \frac{d^{3} l}{(2 \pi)^{3}} \frac{\left(\vec{l}^{2}\right)^{j}}{\vec{l} \cdot \vec{q}}\left\{\theta\left(k_{n}-|\vec{P}-\vec{l}|\right)-\theta\left(k_{p}-|\vec{P}-\vec{l}|\right)\right\} \\
& =\vec{P} \cdot \vec{q} \frac{k_{f}^{2 j+1} \delta}{24 \pi^{2}} I_{j}(s, \kappa)+\mathcal{O}\left(\delta^{3}\right),
\end{aligned}
$$

where $k_{n, p}=k_{f}(1 \pm \delta)^{1 / 3}$ denote the Fermi momenta of neutrons and protons, and $j=0,1$ are exponents of $\vec{l}^{2}$. The expression in the second line follows by substituting $\vec{l} \rightarrow-\vec{l}$ in the second summand. It makes evident that $s p$-mixing can occur only in a medium with unequal neutron and proton densities, $k_{n} \neq k_{p}$. For small isospin asymmetries $\delta$ the available phase-space becomes a (thin) spherical shell of thickness $2 k_{f} \delta / 3$. The net result for the in-medium loop with $s p$-mixing, expanded to linear order in $\delta$, is given in the third line of eq.(58) and it involves the logarithmic functions:

$$
I_{0}(s, \kappa)=\frac{1}{s^{3}}\left\{4 s+\left(s^{2}+\kappa^{2}-1\right) \ln \frac{\left|(s+1)^{2}-\kappa^{2}\right|}{\left|(s-1)^{2}-\kappa^{2}\right|}\right\}, \quad I_{1}(s, \kappa)=8+\kappa^{2} I_{0}(s, \kappa),
$$

where $s=|\vec{P}| / k_{f}$ and $\kappa=|\vec{q}| / k_{f}$ are two dimensionless variables. In fact, the same asymmetric configuration as considered above is present in the calculation of the isospin (or spin) asymmetry energy from the second-order Hartree and Fock diagrams. This explains why $s p$-interference terms proportional to $t_{0} t_{2}$ and $t_{1} t_{2}$ do occur in the expressions $A\left(k_{f}\right)^{(2 \mathrm{nd})}, S\left(k_{f}\right)^{(2 \mathrm{nd})}$ and $S_{n}\left(k_{n}\right)^{(2 \mathrm{nd})}$ written in eqs. $(18,19,21)$. In the case of the energy per particle, $\bar{E}\left(k_{f}\right)$ or $\bar{E}_{n}\left(k_{n}\right)$, one has from the outset just one single Fermi momentum, $k_{f}$ or $k_{n}$, and therefore $s p$-mixing terms cannot emerge for these quantities at second order in many-body perturbation theory.

\section{Appendix B: Interaction through long-range potentials}

In this appendix we derive the first-order Fock and second-order Hartree and Fock contributions as they arise from several long-range interaction potentials. The energy per particle and asymmetry energies are calculated for many-fermion systems with either four or two internal degrees of freedom (e.g. isospin-symmetric nuclear matter or pure neutron matter). Our first candidate is an interaction potential in coordinate-space with an inverse square and an inverse fourth-power dependence on the particle-distance:

$$
\widetilde{V}_{\text {long }}=\frac{\beta_{2}}{\left|\vec{r}_{1}-\vec{r}_{2}\right|^{2}}+\frac{\beta_{4}}{\left|\vec{r}_{1}-\vec{r}_{2}\right|^{4}}
$$

where the parameter $\beta_{2}$ is a length and $\beta_{4}$ is a volume. The calculation of interaction contributions in the fermionic medium requires the Fourier-transform of this long-range potential:

$$
V_{\text {long }}=\frac{2 \pi^{2} \beta_{2}}{\left|\vec{q}_{\text {out }}-\vec{q}_{\text {in }}\right|}-\pi^{2} \beta_{4}\left|\vec{q}_{\text {out }}-\vec{q}_{\text {in }}\right|
$$


where $\vec{q}_{\text {out }}-\vec{q}_{\text {in }}$ is the Galilei-invariant momentum transfer. Taking $V_{\text {long }}$ as an interaction-vertex in momentum-space, the evaluation of the first-order Fock diagram and the second-order Hartree and Fock diagrams in Fig. 1 gives for the four-component system (nuclear matter):

$$
\begin{aligned}
\bar{E}\left(k_{f}\right)= & -\frac{\beta_{2} k_{f}^{2}}{5}+\frac{3 \beta_{4} k_{f}^{4}}{35}+\frac{\beta_{2}^{2} M k_{f}^{2}}{5}\left(2 G-\frac{\pi^{2}}{3}-2 \ln 2\right) \\
+ & \frac{\beta_{2} \beta_{4} M k_{f}^{4}}{35}(4 \ln 2-13-6 G)+\frac{\beta_{4}^{2} M k_{f}^{6}}{315}\left(5 G+\frac{731}{18}-8 \ln 2\right), \\
A\left(k_{f}\right)=S\left(k_{f}\right)= & -\frac{\beta_{2} k_{f}^{2}}{9}+\frac{2 \beta_{4} k_{f}^{4}}{15}+\frac{\beta_{2}^{2} M k_{f}^{2}}{9}\left(2 G+\frac{\pi^{2}}{6}-2 \ln 2\right) \\
& +\frac{4 \beta_{2} \beta_{4} M k_{f}^{4}}{45}(1-3 G+2 \ln 2)+\frac{\beta_{4}^{2} M k_{f}^{6}}{105}\left(5 G+\frac{103}{9}-8 \ln 2\right),
\end{aligned}
$$

where $G=\int_{0}^{1} d x x^{-1} \arctan x=\sum_{n=0}^{\infty}(-1)^{n}(2 n+1)^{-2}=0.9159656$ denotes the Catalan number. Note that $G$ always results from the $d^{3} l$-loop integral belonging to the second-order Fock diagram with two medium-insertions. The analogous calculation in the two-component system (pure neutron matter) gives the following contributions to the energy per particle and the spin asymmetry energy:

$$
\begin{aligned}
\bar{E}_{n}\left(k_{n}\right)= & -\frac{\beta_{2} k_{n}^{2}}{5}+\frac{3 \beta_{4} k_{n}^{4}}{35}+\frac{\beta_{2}^{2} M k_{n}^{2}}{10}\left(4 G-\frac{\pi^{2}}{3}-1-2 \ln 2\right) \\
& +\frac{2 \beta_{2} \beta_{4} M k_{n}^{4}}{35}(\ln 2-1-3 G)+\frac{\beta_{4}^{2} M k_{n}^{6}}{315}\left(5 G+\frac{115}{9}-4 \ln 2\right), \\
S_{n}\left(k_{n}\right)= & -\frac{\beta_{2} k_{n}^{2}}{9}+\frac{2 \beta_{4} k_{n}^{4}}{15}+\frac{\beta_{2}^{2} M k_{n}^{2}}{18}\left(4 G+\frac{\pi^{2}}{6}-1-2 \ln 2\right) \\
& +\frac{\beta_{2} \beta_{4} M k_{n}^{4}}{45}(11-12 G+4 \ln 2)+\frac{\beta_{4}^{2} M k_{n}^{6}}{105}\left(5 G-\frac{65}{36}-4 \ln 2\right) .
\end{aligned}
$$

It is worth to mention that the (singular) $\beta_{2}$-interaction provides an infrared-finite result at second order. Ultraviolet divergences have been treated in dimensional regularization, using the rule: $\int_{0}^{\infty} d l l^{n}=0$. Note that the contributions of second-order Hartree diagrams (with two or three medium insertions) are in nuclear matter a factor 2 larger than in pure neutron matter. The corresponding pieces carry (besides a rational number) the coefficients $\pi^{2} / 3$ and $\ln 2$.

As a second example for a long-range interaction we treat the familiar Van-der-Waals potential:

$$
\widetilde{V}_{\mathrm{vdw}}=\frac{12 B}{\left|\vec{r}_{1}-\vec{r}_{2}\right|^{6}}, \quad V_{\mathrm{vdw}}=\pi^{2} B\left|\vec{q}_{\mathrm{out}}-\vec{q}_{\mathrm{in}}\right|^{3}
$$

where the factor 12 has been introduced in order to get a simpler Fourier-transform. The manybody calculation at first and second order with the cubic interaction-vertex $\pi^{2} B\left|\vec{q}_{\text {out }}-\vec{q}_{\text {in }}\right|^{3}$ leads to the following results for the four-component system:

$$
\begin{aligned}
\bar{E}\left(k_{f}\right)= & -\frac{8 B k_{f}^{6}}{63}+\frac{B^{2} M k_{f}^{10}}{2145}\left(\frac{1115603}{1050}+27 G-128 \ln 2\right) \\
& +\frac{2 B \beta_{2} M k_{f}^{6}}{315}(30 G+53-16 \ln 2)+\frac{B \beta_{4} M k_{f}^{8}}{231}\left(16 \ln 2-7 G-\frac{3133}{30}\right),
\end{aligned}
$$




$$
\begin{aligned}
A\left(k_{f}\right)=S\left(k_{f}\right)= & -\frac{8 B k_{f}^{6}}{21}+\frac{B^{2} M k_{f}^{10}}{297}\left(\frac{613343}{1050}+27 G-128 \ln 2\right) \\
& +\frac{4 B \beta_{2} M k_{f}^{6}}{315}(45 G-8-24 \ln 2)+\frac{4 B \beta_{4} M k_{f}^{8}}{189}\left(16 \ln 2-7 G-\frac{1369}{30}\right),
\end{aligned}
$$

and for the two-component system:

$$
\begin{aligned}
\bar{E}_{n}\left(k_{n}\right)= & -\frac{8 B k_{n}^{6}}{63}+\frac{B^{2} M k_{n}^{10}}{195}\left(\frac{14311}{350}+\frac{27 G-64 \ln 2}{11}\right) \\
& +\frac{4 B \beta_{2} M k_{n}^{6}}{945}(45 G-4-12 \ln 2)+\frac{B \beta_{4} M k_{n}^{8}}{231}\left(8 \ln 2-7 G-\frac{1141}{30}\right), \\
S_{n}\left(k_{n}\right)= & -\frac{8 B k_{n}^{6}}{21}+\frac{B^{2} M k_{n}^{10}}{27}\left(\frac{6701}{350}+\frac{27 G-64 \ln 2}{11}\right) \\
& +\frac{B \beta_{2} M k_{n}^{6}}{315}(180 G-191-48 \ln 2)+\frac{4 B \beta_{4} M k_{n}^{8}}{189}\left(8 \ln 2-7 G-\frac{259}{30}\right) .
\end{aligned}
$$

The expressions in the second lines of eqs.(67-70) stem from the interference terms of the Van-derWaals interaction $V_{\mathrm{vdw}}$ with the (more long-range) $\beta_{2}$ - and $\beta_{4}$-interactions. Finally, one notices that all interaction contributions derived here are in the form of even powers of the Fermi momentum,

$k_{f}$ or $k_{n}$. It is expected that the analytical results compiled in this appendix could be useful for certain condensed matter systems.

\section{Acknowledgements}

I thank G. Colo and J.W. Holt for informative discussions.

\section{References}

[1] M. Bender, P.H. Heenen and P.G. Reinhard, Rev. Mod. Phys. 75, 121 (2003).

[2] J.R. Stone and P.G. Reinhard, Prog. Part. Nucl. Phys. 58, 587 (2007).

[3] K. Moghrabi, M. Grasso, G. Colo, and N. Van Giai, Phys. Rev. Lett. 105, 262501 (2010).

[4] K. Moghrabi, M. Grasso, X. Roca-Maza, and G. Colo, Phys. Rev. C85, 044323 (2012).

[5] K. Moghrabi and M. Grasso, Phys. Rev. C86, 044319 (2012).

[6] K. Moghrabi, M. Grasso, and U. van Kolck, nucl-th/1312.5949.

[7] J.W. Holt, N. Kaiser, and W. Weise, Phys. Rev. C87, 014338 (2013).

[8] L.D. Landau, Sov. Phys. JEPT 3, 101, 920 (1957); Sov. Phys. JEPT. 8, 70 (1959).

[9] J.A. Nolen and J.P. Schiffer, Ann. Rev. Nucl. Sci. 19, 471 (1969).

[10] E. Epelbaum, Prog. Part. Nucl. Phys. 57654 (2006).

[11] E. Epelbaum, H.W. Hammer, and U.-G. Meißner, Rev. Mod. Phys. 81, 1773 (2009).

[12] R. Machleidt and D.R. Entem, Phys. Rep. 503, 1 (2011).

[13] N.M. Hugenholtz and L. Van Howe, Physica 24, 363 (1958).

[14] N. Kaiser, Eur. Phys. J. A48, 148 (2012).

[15] J.W. Holt, N. Kaiser, and W. Weise, Phys. Rev. C87, 014338 (2013).

[16] S. Shlomo, Rep. Prog. Phys. 41, 957 (1978).

[17] B.A. Nolen, W.A. Richter, and R. Lindsay, Phys. Lett. B483, 49 (2000); and refs. therein.

[18] N. Kaiser, Phys. Rev. C69, 034337 (2004).

[19] E. Chabanat, P. Bonche, P. Haensel, J. Meyer, and R. Schaeffer, Nucl. Phys. A653, 231 (1998); and refs. therein. 